\section{Ant Adùress}

on

\section{EXPERIMENTAL POLYURIA.*}

\author{
BY
}

\section{Sir E. SHARPEY-SCHAFER, Hon.M.D., F.R.S., PROFESSOR OF PHYSIOLOGY, UNIVERSITY OF EDINBURGH.}

SrNce experimental polyuria depends upon injury or disease of the pituitary body, or, as some hold, of the nervous system in the immediate neighbourhood of the pituitary, it will be well first of all to draw attention to the main features of structure of the pituitary which might have a bearing on the subject.

\section{Anatomical Considerations.}

The pituitary lies at the base of the brain in the sella turcica and is connected with the third ventricle by a stalk, which is hollow as far as the gland; the hollow extends in some animals far into the interior. This hollow stalk is the infundibulum. Its wall is formed by a prolongation of the nervous tissue of the floor of the ventricle. This tissue expands into the posterior part of the gland, forming a solid mass of neuroglia-the pars nervosa or pars infundibularis. Nerve cells are absent. It is composed mainly of neuroglia with a few nerve fibres. Surrounding and enclosing the stalk is a prolongation of the glandular substance, which spreads from the stalk over the neighbouring part of the brain, especially over the tuber cinereum this forms the pars tuberalis. A cross-section of the stalk shows a complete ring of pars tuberalis encircling the nervous tissue of the stalk proper. Anterior to and largely enclosing the pars nervosa, the pituitary is formed of a mass of epithelium cells of granular appearance. This mass constitutes what is known as the pars anterior.

It is the most vascular part of the gland, and when exposed in the living animal is conspicuous by its pronounced yellowish-red colour.

In most animals there is a cleft in the middle of the gland which separates the organ into two !obes, a larger anterior and a smaller posterior. In the adult human subject the cleft is generally obliterated. In front of the cleft is the pars anterior, which forms the anterior lobe of the gland; behind it is the pars nervosa, but this part is covered by an epithelium layer which is intimately adherent to it, termed the pars intermedia. The pars nervosa plus the pars intermedia together form the posterior lobe. The organ is easily separable into the two lobes by splitting it across in the line of the cleft. The cleft is the remains of the original tube which is formed in the early embryo from an outgrowth of the buccal ectoderm. The tube communicates at first with the buccal cavity, although the communication becomes obliterated later.

\section{Pars Anterior.}

This composes by far the larger portion of the gland, and is formed of epithelium separated by connective tissue stroma. The epithelium is arranged in cord-like or isolated masses of cells. These masses are usually solid, but sometimes the cells surround a vesicle filled with " colloid," which is no doubt secreted by the cells. Very numerous thin-walled sinus-like blood vessels traverse this part of the gland. They lie in intimate relation to the ceils, the secretion of which is probably passed directly into them. The great vascularity of the pars anterior is very apparent in a photograph of an injected gland.

\section{Pars Tuberalis.}

The larger portion of this forms an extension of the gland forwards over the tuber cinereum. This portion corresponds with what was termed by Herring the "tongue-like process." He described it as an extension of the pars intermedia, but alike in structure and development it is distinct both from the pars intermedia and pars anterior. Its cells are basophil, not oxyphil; they are for the most part

\footnotetext{
* Address to the Inter-State Post-Graduate Assembly of America. Friday
} June 19th, 1925 . arranged around colloid-containing vesicles somewhat like those of the thyroid. In vascularity the pars tuberalis closely resembles the pars anterior, so that in a photograph from an injected specimen the two parts are almost indistinguishable. Moreover, its capillaries have a sinusoid character like those of the pars anterior. This extreme vascularity renders it probable that it has an important function to fulfil.

As we have seen, the pars tuberalis extends around the stalk of the pituitary in the form of a ring, which spreads over the adjacent part of the base of the brain, especially over the tuber cinereum.

\section{Pars Intermedia.}

The pars intermedia lies immediately behind the intraglandular cleft, covering the anterior surface of the pars nervosa, around which in many animals it sends a sheathlike prolongation for a variable distance. Here and there it extends into the substance of the pars nervosa. These extensions may become cut off from the rest and appear as islets of pars intermedia in the thickness of the pars nervosa.

The cells of the pars intermedia are smaller than those of the pars anterior. 'They resemble the cells of the pris tuberalis in being devoid of the coarse oxyphil and basophil granules which characterize those of the pars anterior. Here and there they may be seen surrounding circular vesicles occupied by colloid. Occasionally this is in considerable quantity and the vesicles are correspondingly large; on the other hand, it is sometimes absent.

Besides such colloid as is contained within the vesicles the pars intermedia often exhibits globular masses of a similar substance of varying size not enclosed within vesicles (Herring's bodies). Some of these masses contain the remains of a nucleus, and have evidently been produced by a chemical transformation of the protoplasm of some of the epithelium cells. The masses are sometimes hyaline, sometimes granular. They are traceable into and through the pars neivosa, and can even be seen passing into the infundibulum of the third ventricle owing to its extension into the pars nervosa. It appears that they may undergo a further clremical transformation in the passage, for, according to Herring, extracts of pure pars intermedia and of pure pars nervosa show profound differences in their phy'siological effects.

'The passage of colloid masses from the pars intermedia into and through the pars nervosa to be ultimately discharged into the third ventricle and thus into the cerebrospinal fluid was discorered by Herring. He concluded that the substance of these masses represents the secretion of the pars intermedia, which is thus passed into the ventricle. Herring's observations have been abundantly corroborated by subsequent workers who have used the same methods of fixation and staining. It is probable that the pars nerrosa owes the physiological activity of its extracts to this material.

Confirmatory evidence that the active principles of the pituitary body can pass into the cerebro-spinal fluid has been furnished by various observers. Harvey Cushing and Groetsch found that the cerebro-spinal fluid when reduced in amount by evaporation will produce some of the characteristic effects of extracts of the posterior lobe; but the main characteristic effect of these extracts-that, namely, of producing extreme contraction of uterine muscle-was unknown at the time of their experiments. More recently evidence has been accumulating to show the existence of this uterus-contracting or oxytocic principle in cerebrospinal fluid. Douglas Cow, working with Dixon at Cambridge, was the first to show that duodenal extract if injected into the blood will produce after a certain interval unmistakable evidence, as tested with a uterine cornu, of the presence of the oxytocic principle in the cerebro-spinal fluid. More recently Dixon and Marshall of Cambridge have shown that ovarian extract is also a powerful stimulator for the pituitary, causing the oxytocic principle to be passed into the cerebro-spinal fluid. This only occurs in the absence of the corpus luteum from the ovary. Extracts which are made from an ovary during the greater part of pregnancy do not produce this effect; 
but if an extract is made at the end of pregnancy and after the corpus luteum has undergone involution, a marked effect is produced, so that a few drops of cerebrospinal fluid will cause strong contraction of the uterus. It is interesting to correlate this effect with the well known therapeutic influence of pituitary extracts upon the parturient uterus. The effect is not a direct one of duodenal extract or of ovarian extract, since these are in themselves inert so far as the uterus and diuresis is concerned. Moreover, the action will not occur if the pituitary has been removed. Confirmatory observations have been made by Trendelenburg, who has shown that the cerebro-spinal fluid of the dog contains an oxytocic principle which is absent from it if the pituitary has been previously extirpated or its stalk severed.

We have seen that the pars nervosa contains the colloid masses of Herring which are passed into it from the pars intermedia. They can be observed to make their escape into the extension of the third ventricle towards the pituitary, and, as Herring has shown, are greatly increased in number after thyroidectomy. Mott has made a similar observation in a case of myxoedema occurring in man.

Vascular Supply.

With regard to the vascular supply of the pituitary, Goetsch has shown that in the dog it is supplied by a large number of arterioles derived from the circle of Willis and from a communicating branch uniting the internal carotids. The arterioles converge towards the infundibulum and are conveyed to the gland along its stalk. Most of the blood is distributed to the pars anterior. The venules, which are also very numerous, pass at first to the stalk and thence diverge and form small veins radiating outwards to enter a venous circle roughly corresponding with the arterial circle. It is clear from this description that severance of the stalk would entail cutting off the main blood supply of the gland. There appear to be no true lymph vessels in the gland. Any lymph which is formed within it must find its way through the space between the neuroglial fibres into the third ventricle or its extension.

\section{Nerve Supply.}

Very little is known about the nerve supply of the pituitary. Fibres have been described as passing along the stalk from sympathetic branches on the internal carotids. Such nerves are doubtless derived from the superior cervical ganglion. They are non-myelinated and are probably mainly distributed to the blood vessels. But myelinated nerve fibres have also been seen ending in ramifications amongst the cells of the pars anterior. Apparently there are no nerve cells in the pars nervosa.

\section{Physiology.}

It is not necessary to describe all the physiological effects which are produced by extracts of the posterior lobe of the pituitary body; but in connexion with experimental polyuria it is important that certain facts should be pointed out. The first is that extracts of the posterior lobe of the pituitary when injected into the vascular system cause a great rise of blood pressure with contraction of arterioles and increased force of the heart beats. Sometimes the pressor effect is preceded by a fall of blood pressure, and if a second dose is administered there is always a fall and no subsequent rise. This appears to be due to the fact that there are two antagonistic principles in such extracts, one causing elevation of blood pressure and the other depression. The principle which causes fall of blood pressure is, if not identical with, at any rate closely allied to histamine. This is shown not only by the effect on the aortic pressure but also by that which is produced upon pulmonary pressure. It was observed by Wiggers that an urdinary extract of posterior lobe of pituitary body is apt to cause, along with the raising of the aortic pressure, a lowering of pulmonary pressure. But we have found that if the histamine-like substance is first completely extracted by absolute alcohol, only a pressor effect is obtained, in both aortic and pulmonary systems. The rise of blood pressure is due to general contraction of arteries; but those of the kidney, in place of being contracted, are relaxed by the extract.
Diuretic Action.

For our present subject this exception is of great importance since the dilatation is correlated with an increase of secretion of urine, often very marked. This effect may be preceded by a slight contraction and the increase of urine by a temporary diminution. The increased secretion might be explained by the increased flow of blood through the kidney vessels due to the circumstance that they undergo dilatation while the other systemic arteries are contracting, so that the blood pressure in the glomeruli is raised; but that it is to a certain extent caused by a specific effect of one of the pituitary principles, and is not merely a mechanical result of increased blood pressure, is probable from the fact that it may occur in the absence of any obvious rise in blood pressure. Moreover, if there is at first a rise in blood pressure the increased rate of flow of urine is maintained even after the blood pressure has come back to its original level. And the effect of a second dose of the extract is again to produce an increase in the flow of urine, although the blood pressure no longer rises but falls, and although the kidney volume may now be unaffected: This would seem to indicate that the active principle affects not only the blood vessels of the kidney but also its secreting cells, which it renders more permeable; and the effect must be looked upon as caused by a chemical action on the renal cells similar to that of secretin on the cells of the pancreas. In this respect the action of the diuretic principle is comparable to that of those drugs which act as specific diuretics upon the kidney as distinguished from those which produce diuresis by merely increasing the general blood pressure.

The diuretic action of the pituitary is not antagonized by atropine. This may be taken as a sign that it does not act through nerves or nerve endings, but directly upon the renal cells. In this respect also it resembles the action of secretin upon the pancreas.

The ordinary extracts of the posterior lobe of the pituitary with which we are now dealing are commonly made with water or Ringer's solution, but if an alcohol extract is employed not only is a depressor effect obtained upon the circulation instead of the pressor effect, but the urinary secretion is diminished in place of being increased.

Herring states that the diuresis only occurs when extracts of pars nervosa are employed. If extracts are made from pars intermedia or pars anterior no such effect is obtained. No one has yet succeeded in determining whether extracts of pars tuberalis contain the diuretic principle.

\section{Antidiuretic Action.}

Besides the diuretic action of extracts of posterior lobe, these are found in certain circumstances to bring abcut exactly the opposite condition. When intravenous injections of pituitary extract are made, there is, as we have seen, at once a very temporary diminution of flow of urine followed by a prolonged increase. On the other hand, with subcutaneous or intramuscular injection, especially if polyuria is already present, a great diminution in tho secretion is produced. The antidiuretic action was first noticed in clinical cases of diabetes insipidus, in which it has a remarkable, indeed specific, action in controlling the excretion of water by the kidneys and bringing it down to a normal level. To keep it at that the injections require to be repeated from time to time. In normal individuals the effect is less striking.

Most experiments on the antidiuretic action of pituitary extracts have been made on water diuresis - that is, the polyuria which is produced by drinking large quantities of water. If pituitary extract is administered previously to taking large quantities of water, a considerable delay of. the water diuresis occurs, and during this there, are nervous symptoms, due doubtless to the accumulation of water in the system, which are sometimes of a serious character. Molitor and Pick find that the toxic effects which may be caused by pituitary when a large excess of water is administered can be at once removed by the administration of urea, which probably acts by removing. the excess of water from the tissues in which it has been stored and causing its rapid excretion by the kidneys. They conclude that the antidiuretic effect has nothing to do with the renal vessels or with the renal cells. but is an effect 
on the tissues, perhaps also on the capillaries, and that one of the main functions of the pituitary is to determine the amount of water the tissues can hold.

\section{Polyuria as a Result of Pituitary Lesions.}

A very common effect of experimental injury of the pituitary is polyuria. Considering that extracts of the posterior lobe of the pituitary produce diuresis when injected into the blood, it seems possible that injury of the lobe without removal may produce irritation either of the pars intermedia or of the pars tuberalis, and thus cause the outpouring of an increased secretion from these parts of the gland. The polyuria which is often found to accompany fracture of the base of the skull may be due to a concomitant injury of the pituitary. Mere manipulation of the posterior lobe without causing any obvious lesion will produce polyuria, but exposure of the gland by lateral operation without manipulation or injury has no such effect. In one experiment on a dog of injury of the posterior lobe by a touch with the electric cautery I found the amount of urine secreted to rise from $40 \mathrm{c.cm}$. a day to $230 \mathrm{c.cm}$. For nineteen days it showed an average of $119 \mathrm{c.cm}$. In another experiment the amount of urine rose from $110 \mathrm{c.cm}$., the average for the preceding eleven normal days, to $182 \mathrm{c.cm}$. for the eleven days immediately subsequent to the operation, and on some of these days it reached much more than this, attaining as much as 266 c.cm. Many others have recorded cases of temporary or permanent polyuria produced by an injury to the pituitary body or to its stalk. It is true that in some of these there were injuries to the adjacent part of the brain, but this was not the case with all of them. Cushing obtained diuresis by stimulation of the cervical svmpathetic or of its superior ganglion, and, according to him and his fellow workers, puncture of the pituitary may give as definite results regarding glycosuria and polyuria as Bernard's puncture of the fourth ventricle.

Most of the experiments the results of which in causing polyuria, with or without glycosuria, have been recorded have taken no account of the pars tuberalis. This part cannot easily be studied separately by reason of the fact that it is intimately united with the base of the brain, over which it spreads for some little distance. It may be that this portion of the pituitary is responsible for the polyuria which is frequently associated with injury of the gland; and either injury to the pars tuberalis or interference with the blood supply of the gland may be accountable for the results which have been recorded by Camus and Roussy. These observers have obtained in the dog, cat,'and rabbit, but not with the same facility in all; polyuria and glycosuria from injuries by puncture or cautery to the floor of the third ventricle (tuber cinereum) in the immediate vicinity of the pituitary-apparently, as they state, without injury to the gland itself. They consider that in all cases the polyuria is due to the brain lesion and not to any effect on or through the pituitary, which can, they state, be removed previously without affecting the results. But it may be stated that it is not possible to remove the pars tuberalis without injuring the base of the brain. Camus and Roussy even ascribe the dystrophia adiposo genitalis and all the other effects which have been put down to lesions of the pituitary to injuries of the tuber cinereum.

Similar results have been recorded by Bailey and Bremner as being produced in the dog by a puncture by a probe in the base of the brain in the neighbourhood of the pituitary, without in any way directly injuring the gland, the integrity of which was verified histologically (although the pars tuberalis is not mentioned). Polyuria resulted from even the smallest puncture. It is significant that it began, not immediately, but within the first two days and lasted six to eight days or longer. When it was persistent the symptoms known as cachexia hypophyseopriva, genital atrophy, and adiposity were also manifested; all suggestive of pituitary insufficiency. The persistent polyuria when it occurred had all the characteristics of diabetes insipidus in man; such as concentration of urine when intake of water was restricter, excessive polvuria when chlorides were injected, diminution of urine when posterior lobe extract was injected, etc. According to these authors, polyuria is never produced by lesions outside the peri-infundibula region, but nevertheless in their opinion it is not caused by the agency of the pituitary directly or indirectly.

Although there can be no question as to the facts recorded by the above-mentioned authors, it is difficult, in view of the numerous observations on the effects of lesions confined to the gland and of the administration of extracts of the gland, to believe that most, if not all, of the symptoms described have not been produced through the agency of the pituitary. It is difficult to believe that a minute lesion of the floor of the third ventricle, very different in structure and nerve connexions from the floor of the fourth ventricle (in fact of simpler structure and with fewer nerve connexions than any other part of the brain, and having no trace of glandular structure except that which is derived from the subjacent pituitary body), should produce per se such far-reaching results as these described, and litherto attributed to disturbances in the activity of the pituitary itself. It seems a more rational conclusion that the perihypophyseal iesions effected by Camus and Roussy and by Bailey and Bremner produce their effects by disturbing the normal functions of the gland, either through its vascular supply or perhaps through hitherto unrecognized nervous connexions; or by interfering with the passage of products of the secretion of the pars intermedia into the ventricle, rather than by direct nervous action.

Most of the discussion has turned on the question of the production of polyuria from lesions of the base of the brain in the perihypophyseal region. The fact that polyuria is produced by such lesions even when the kidneys are completely denervated is a clear indication that the cause of the polyuria is humoral rather than nervous. 'This and the fact that it does not make its appearance until the next day or the day after are suggestive of an indirect action through the pituitary rather than of a direct effect from the brain lesion, which, as in the case of Bernard's puncture of the fourth ventricle, would produce its effects immediately.

\section{THE PRESENCE POST MORTEM OF NITRIC- OXIDE-HAEMOGLOBIN :}

\section{ITS CLINICAL AND MEDICO-LEGAL SIGNIFICANCE.}

BY

H. A. L. BANHAM, L.R.C.P., J. S. HALDANE, M.D., F.R.S., AND THOMAS SAVAGE, M.B.

The case on which the present paper is mainly founded was one in which there is every reason for believing that postmortem appearances which simulated very closely those of carbon monoxide poisoning were due to the formation of nitric-oxide-haemoglobin after death.

A man employed at a colliery in stoking the boiler furnaces became ill on his way home after a full day's work. He became faint and unable to stand. Even after a rest he was unable to walk more than a few yards; and he was brought home in a car. $H$ is skin was very cold and he was very feeble. During the night he was bringing up blood-stained sputum. He was seen next day by his doctor, who found that his pulse was feeble, his breathing heavy, his temperature normal, and that he was complaining of a feeling of compression in the chest. Carbon monoxide poisoning feeling of compression in the chest. Carbon monoxide poisoning was suspected, and he was kept in bed; but the symptoms con-
tinued, including the blood-stained sputum. On the ninth day tinued, including the blood-stained sputum. On the ninth day
after his illness began he was getting worse, and was sent in a taxi to the Beckett Hospital, Barnsley; with a view to treatment by transfusion of blood. When seen on arrival by one of us (T. S.) he was very cyanosed and evidently moribund, and he died within ten minutes.

A post-mortem examination (by T.S.) was made within a few A post-mortem examination (o the cause of death were as hours. The only signs pointing to the cause of death were as follows. The lungs contained much frothy blood-stained liquid, and showed patches of pneumonia, though they floated in water. There was also a good deal of fluid in the pleural and pericardial The whole of the blood, in whatever part of the body, including heart, spleen, kidneys, muscles, and lungs, had a red or pink heart, spleen, kifferent from the dark colour normally seen at colour encutting into these organs in post-mortem examinations.

This appearance was thus identical with that in a case where death from carbon monoxide poisoning has occurred while the victim was breathing the poisonous air or directly afterwards. On dilution with water the blood gave a pink 